\title{
195. NUEVAS LOCALIDADES DE ODONTITES VISCOSUS SUBSP. GRANATENSIS (BOISS.) BOLLIGER (SCROPHULARIACEAE)
}

\author{
Julián Manuel FUENTES CARRETERO ${ }^{1 *}$ \& Jose Antonio ALGARRA ÁVILA²
}

Recibido el 26 de marzo de 2015, aceptado para su publicación el 30 de abril de 2015

New records of Odontites viscosus subsp. granatensis (Boiss.) Bolliger (Scrophulariaceae).

Palabras clave. Odontites, corología, ecología, sureste de la Península Ibérica, Andalucía, Sierra Nevada, Scrophulariaceae.

Key words. Odontites, chorology, ecology, southeastern Spain of Iberian Peninsula, Andalusia, Sierra Nevada, Scrophulariaceae.

Odontites granatensis Boiss. fue descrito inicialmente como especie (Boissier, 1838), aunque tras revisiones y estudios sucesivos fue considerado como subespecie de Odontites viscosus (Bolliger, 1996; Bolliger et al., 1992; Rico \& Romero, 2002; Rico, 2009, 2011). Se trata de uno de los taxones endémicos más amenazados de Sierra Nevada (Granada). En épocas recientes solo se tenía constancia de una población que no supera $1 \mathrm{~km}^{2}$ de superficie ocupada, en la zona caliza noroccidental de Sierra Nevada (Blanca et al., 2001; Gutiérrez et al., 2004). Además, dicha población fue parcialmente sepultada en 1994, debido al movimiento de tierras durante las obras de acondicionamiento de la Estación de esquí de Sierra Nevada, para el Campeonato Mundial de Esquí Alpino celebrado en 1996.

En lo que sigue, la nomenclatura empleada y la verificación corológica son las que se incluyen en Flora Iberica (Rico, 2009) y Flora Vascular de Andalucía Oriental (Rico, 2011). El material recolectado está depositado en el herbario de la Universidad de Granada (GDA).

Existen cuatro citas bibliográficas antiguas (Quézel, 1953), todas ellas pertenecientes al actual Parque Nacional de Sierra Nevada; tres de ellas en el barranco del río Guarnón (lugar dónde se encuentra una de las poblaciones localizadas por nosotros) y otra próxima a Laguna Larga (localidad muestreada exhaustivamente sin éxito). Además, hay un pliego testigo (GDA 9237) colectado en la zona de Terreras Azules, a $2.550 \mathrm{~m}$ de altitud, localidad relativamente cercana a la descubierta ahora en el barranco de río Seco.

Las dos nuevas poblaciones quedan incluidas en el ámbito del Espacio Natural de Sierra Nevada (fig. 1). La primera en la parte noroccidental, más concretamente en el barranco del río Guarnón, citada por Quézel (1953) y confirmada ahora (GDA 61400); se sitúa entre 2.100 y $2.350 \mathrm{~m}$ de altitud, constituida por diversos núcleos con 5 a 38 ejemplares, con un número total de individuos que varió entre 392 y 513, según los muestreos y prospecciones realizados entre los años 2009 y 2014, y un área de ocupación de 0,2 $\mathrm{km}^{2}$. La segunda, se sitúa en la parte suroeste (GDA 9237, GDA 61398 y GDA 61399), en el barranco de Río Seco, entre 2.440 y 2.500 $\mathrm{m}$ de altitud, constituida por dos pequeños núcleos, de 24 y 30 ejemplares, con un total de 54 individuos, según el muestreo realizado en el año 2012 y un área de ocupación de poco más de $0,0000005 \mathrm{~km}^{2}$.

Con estos nuevos datos Odontites viscosus subsp. granatensis, la extensión de presencia es 
de $3,59 \mathrm{~km}^{2}$. Respecto al número de individuos, al tratarse de una especie anual, presenta fluctuaciones extremas interanuales.

Mientras que en la localidad conocida hasta el momento Odontites viscosus subsp. granatensis vegeta en el ambiente del sabinar calcícola oromediterráneo, en comunidades de Daphno hispanicae-Pinetum sylvestris y Astralago boissieri-Festucetum hystricis (Martínez Lirola et al., 1999), en las dos nuevas localidades aportadas ahora, los individuos prosperan en sustratos ultrabásicos de origen metamórfico, de $\mathrm{pH}$ alcalino, materiales muy ricos en mármoles, serpentinas, gneises, etc, que hacen posible el establecimiento de especies típicamente basófilas, ya que los requerimientos ecológicos son los adecuados para su establecimiento, como ocurre con otros taxones como Artemisia alba subsp. nevadensis (Willk.) Blanca \& C. Morales en la localidad del barranco del río Guarnón y Andryala agardhii DC. en el barranco de Río Seco. Ambas localidades se encuentran en el piso oromediterráneo, en pastizales psicroxerófilos, entre lastonares y matorrales de porte pulvinular (piornales), donde se hallan como acompañantes Thymus serpylloides Bory subsp. serpylloides, Koeleria crassipes Lange subsp. crassipes, Agrostis nevadensis Boiss., Dactylis glomerata subsp. juncinella (Bory) Stebbins \& Zohary, Hormathophylla spinosa (L.) P. Küpfer, Eryngium bourgatii Gouan, Linaria aeruginea subsp. nevadensis (Boiss.) Malag., Nepeta nepetella subsp. laciniata (Willk.) Aedo, Campanula rotundifolia subsp. willkommii (Witasek) Blanca, Coincya monensis subsp. nevadensis (Willk.) Leadlay, Biscutella glacialis (Boiss. \& Reut.) Jord., Sempervivum minutum (Willk.) Pau, Sedum annuum L., Genista versicolor Boiss., Acinos alpinus subsp. meridionalis Nyman, Cerastium alpinum subsp. nevadense (Pau) Martínez Parras \& Molero Mesa.

Es importante señalar que en estas dos nuevas localizaciones los individuos presentan

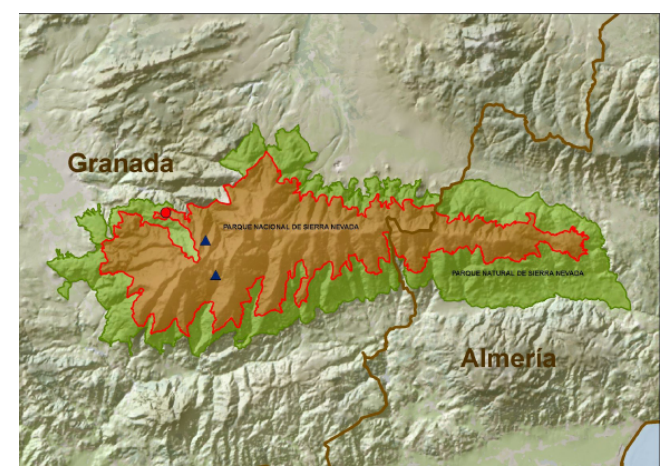

Figura 1. Distribución conocida de Odontites viscosus subsp. granatensis. Círculo: localidad previamente conocida; triángulos: nuevas citas. Known distribution Odontites viscosus subsp. granatensi. Red circle, previously known locations; blue triangles, new appointments.

corolas amarillas (fig. 2), mientras que en la población clásica las corolas son de color granate/púrpura (fig. 3). La existencia de unos individuos con corolas de color granate y otros de corolas amarillas ocurre igualmente en Odontites viscosus subsp. asturicus M. Laínz, subespecie endémica de la Cordillera Cantábrica y Montes de León, y en las otras subespecies de $O$. viscosus de área más amplia (Rico, 2009).

En cuanto al grado de amenaza, la última evaluación realizada procede del Atlas de Flora Vascular Amenazada de España (Gutiérrez et al., 2004): En Peligro Crítico [CR A2abde; $\mathrm{B} 1 \mathrm{ab}(\mathrm{iii}, \mathrm{v})+2 \mathrm{ab}(\mathrm{iii}, \mathrm{v})]$. La normativa que 10 protege incluye en primer lugar la de tipo indirecto, por habitar en el interior del Parque Nacional de Sierra Nevada (Anónimo, 1999) y en segundo lugar la de tipo directo, donde aparece en el Listado de Especies Silvestres en Régimen de Protección Especial (Anónimo, 2011), en el Catálogo Andaluz de Especies Amenazadas (Anónimo, 2012b) y en el Plan de Recuperación y Conservación de Especies de Altas Cumbres de Andalucía (Anónimo, 2012a), con la categoría En Peligro de Extinción en estos dos últimos. 


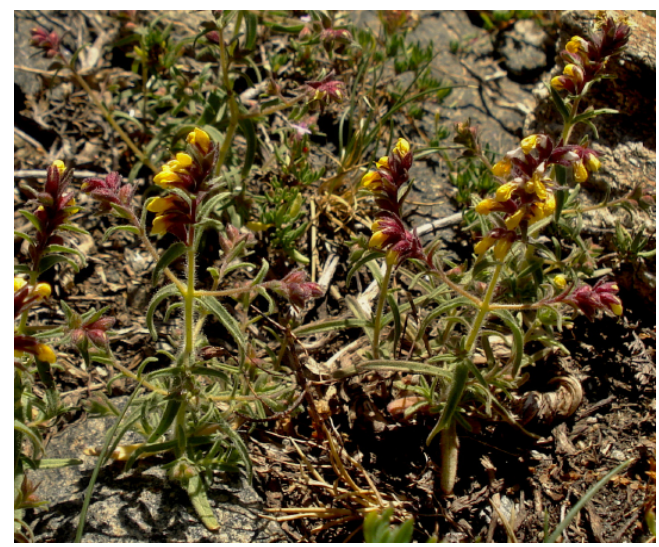

Figura 2. Odontites viscosus subsp. granatensis, Capileira (Granada), Sierra Nevada, bco. De Río Seco, 20/08/2009.

Gracias al hallazgo de estas nuevas localidades, así como a los esfuerzos de conservación realizados en la población clásica, englobados en las iniciativas recogidas en el Plan de Recuperación y Conservación de Especies de Altas Cumbres de Andalucía (Anónimo, 2012a), mejora ligeramente el diagnóstico sobre su estado de conservación, aunque la reevaluación de la categoría de amenaza con los criterios UICN (2012) lo mantiene en la máxima categoría de amenaza: CR B1b(ii,iii)c(iv)+2b(ii, iii)c(iv). Teniendo en cuenta su especificidad ecológica, las prospecciones exhaustivas realizadas y la escasez de zonas potencialmente óptimas para su desarrollo, es poco probable la aparición de nuevos núcleos poblacionales. Por tanto, se estima urgente seguir impulsando el Plan de Recuperación y Conservación de Especies de Altas Cumbres de Andalucía, controlando la predación por parte de grandes ungulados, tanto domésticos (bovino y ovino) como silvestres (Capra pyrenaica Schinz subsp. hispanica Schimper); para ello, se estima como una medida muy efectiva el levantamiento de pequeños cercados de exclusión temporal, que permitan la dinámica natural propia de sus comunidades vegetales y, a la vez, protejan

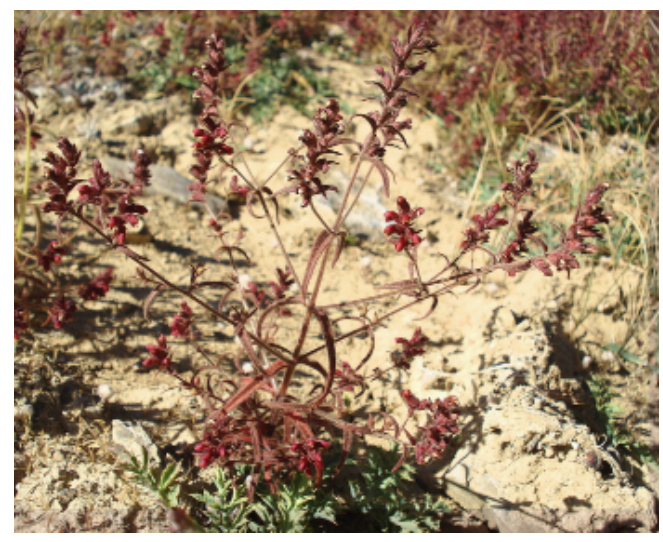

Figura3. Odontites viscosus subsp. granatensis, Monachil (Granada), Sierra Nevada, Collado de las Sabinas, 11/08/2007.

las pequeñas poblaciones en los momentos más críticos de su ciclo vital. En algunas de las localidades señaladas, se han observado los individuos ramoneados hasta tal extremo que algunos años no llega a fructificar ni un sólo individuo.

\section{Material estudiado}

ESPAÑA. Granada. 30SVF7098, Capileira, Sierra Nevada, bco. de Río Seco, suelos esquistosos, $2.500 \mathrm{~m}, 02 / 09 / 2009, J$. Fuentes \& J. Algarra (GDA61398). 30SVF7098, Capileira, Sierra Nevada, bco. de Río Seco, suelos esquistosos, $2.500 \mathrm{~m}, 20 / 08 / 2009, \mathrm{~J}$. Fuentes (GDA 61399). 30SVG6804, Güéjar Sierra, Sierra Nevada, bco. del Guarnón, entre el piornal aclarado, pastizales, lastonares y piornales, sobre sustratos ultrabásicos, 2.100-2200 m, orientación NE, 27/08/2010 02/10/2014, J. Fuentes, J. Algarra \& D. Pinto (GDA 61400). 30SVF6997, Capileira, Sierra Nevada, Tajos Colorados, pedregales, 2.550 m, 22/07/1979, J. Molero Mesa (GDA 9237).

AGRADECIMIENTOS. A Gabriel Blanca, Daniel Pinto, la Consejería de Medio Ambiente y Ordenación del Territorio, el Espacio Natural de 
Sierra Nevada, así como al personal del Herbario de la Universidad de Granada, por sus valiosas ayudas prestadas.

\section{BIBLIOGRAFÍA}

ANÓNIMO -1999- Ley 3/1999, de 11 de enero, por la que se crea el Parque Nacional de Sierra Nevada. BOE 11: 1512-1525.

ANÓNIMO -2011- Real Decreto 139/2011, de 4 de febrero, para el desarrollo del Listado de Especies Silvestres en Régimen de Protección Especial y del Catálogo Español de Especies Amenazadas. BOE 46: 20912-20951.

ANÓNIMO -2012a- Acuerdo de 13 de marzo de 2012, del Consejo de Gobierno, por el que se aprueban los planes de recuperación y conservación de determinadas especies silvestres y hábitats protegidos. BOJA 60: 164-207.

ANÓNIMO -2012b- Decreto 23/2012, de 14 de febrero, por el que se regula la conservacion y el uso sostenible de la flora y la fauna silvestres y sus hábitats. BOJA 60: 114-163.

BLANCA, G., M.R. LÓPEZ ONIEVA, J. LORITE, M.J. MARTÍNEZ LIROLA, J. MOLERO, S. QUINTAS, M. RUÍZ GIRELA, M.A. VARO \& S. VIDAL -2001- Flora amenazada y endémica de Sierra Nevada. Universidad de Granada. Consejería de Medio Ambiente. Junta de Andalucía, Granada. 407 pp.

BOISSIER, P. E. -1838- Elenchus plantarum novarum minusque cognitarum quas in itinere hispanico. Typographia Lador et Ramboz, Genevae. 94 pp.

BOLLIGER, M. -1996- Monographie der Gattung Odontites (Scrophulariaceae) sowie der verwandten Gattungen Macrosyringion, Odontitella, Bornmuellerantha und Bartsiella. Willdenowia 26: 37-168.

BOLLIGER, M., R. BOLLIGER \& G. HEUBL -1992- Geographische Rassen von Odontites viscosus (L.) Clairv. Flora 186: 195-223.

GUTIÉRREZ, L., G. BLANCA, \& J. LORITE -2004- Odontites granatensis Boiss. En: A. Bañares, G. Blanca, J. Güemes, J.C. Moreno \& S. Ortiz (eds.), Atlas y Libro Rojo de la Flora Vascular Amenazada de España, pp. 418-419. Dirección General de Conservación de la Naturaleza, Madrid.
MARTÍNEZ LIROLA, M. J., G. BLANCA \& J. MOLERO -1999- Odontites granatensis Boiss. En: G. Blanca, B. Cabezudo, J.E. HernándezBermejo, C.M. Herrera, J. Molero Mesa, J. Muñoz \& B. Valdés (eds.), Libro Rojo de la Flora Silvestre Amenazada de Andalucía. Tomo I: Especies en Peligro de Extinción, pp. 206-209. Consejería de Medio Ambiente, Junta de Andalucía, Sevilla.

QUÉZEL, P. -1953- Contributions à l'étude phytosociologique et botanique de la Sierra Nevada. Mem. Soc. Broteriana 9: 32-45.

RICO, E. \& T. ROMERO -2002- Odontites Ludw. En: J. A. López-Sáez, P. Catalán \& L. Sáez Goñalons (eds.), Plantas parásitas de la Península Ibérica e Islas Baleares, pp. 220-245. Ediciones Mundi-Prensa, Madrid.

RICO, E. -2009- Odontites Ludw. En: C. Benedí, E. Rico, J. Güemes \& A. Herrero (eds.), Flora iberica 13: 473-495. Real Jardín Botánico. CSIC, Madrid.

RICO, E. -2011- Odontites Ludw. En: G. Blanca, B. Cabezudo, M. Cueto, C. Morales Torres \& C. Salazar (eds.), Flora vascular de Andalucía oriental, pp. 1281-1283. Universidades de Almería, Granada, Jaén y Málaga. Granada.

UICN -2012-Categorías y criterios de la lista roja de la UICN: Versión 3.1. Segunda edición. UICN, Gland, Suiza y Cambridge, Reino Unido. 34 pp.

Dirección de los autores: ${ }^{1} \mathrm{C} /$ Castillo 5, bajo f, 18152 La Zubia (Granada). ${ }^{2} \mathrm{C} /$ Mirlo 27, Maracena (Granada). *Autor para correspondencia: fuentescarretero@hotmail.com 